\title{
Development of an Implementation Intervention Using Intervention Mapping to Increase Mammography Among Low Income Women
}

\author{
Linda Highfield $^{1 *}$, Melissa A. Valerio ${ }^{1,2}$, Maria E. Fernandez ${ }^{3}$ and \\ L. K. Eldridge-Bartholomew ${ }^{3 \dagger}$
}

\begin{abstract}
${ }^{1}$ Department of Management, Policy and Community Health Practice, UTHealth School of Public Health, Houston, TX, United States, ${ }^{2}$ Department of Health Promotion and Behavioral Sciences, UTHealth School of Public Health, San Antonio, TX, United States, ${ }^{3}$ Department of Health Promotion and Behavioral Sciences, UTHealth School of Public Health, Houston, TX, United States
\end{abstract}

\section{OPEN ACCESS}

Edited by:

Shane Andrew Thomas,

Shenzhen International Primary Healthcare Research Institute, China

Reviewed by:

Miodraga Stefanovska-Petkovska, Universidade de Lisboa, Portugal Nobhojit Roy,

Karolinska Institutet (KI), Sweden

Paolo Giorgi Rossi,

Azienda Sanitaria Unità Locale di

Reggio Emilia, Italy

*Correspondence:

Linda Highfield

Linda.d.highfield@uth.tmc.edu

${ }^{\dagger}$ Deceased

Specialty section: This article was submitted to

Public Health Education and

Promotion,

a section of the journal

Frontiers in Public Health

Received: 05 March 2018 Accepted: 28 September 2018 Published: 26 October 2018

Citation: Highfield L, Valerio MA, Fernandez ME and Eldridge-Bartholomew LK (2018)

Development of an Implementation Intervention Using Intervention Mapping to Increase Mammography Among Low Income Women.

Front. Public Health 6:300. doi: 10.3389/fpubh.2018.00300
Background: Although much work has begun to elucidate contextual factors influencing implementation, the specific processes that facilitate and hinder adoption, implementation, and maintenance of evidence-based interventions (EBIs) in clinical settings remains poorly understood. Intervention Mapping (IM) is a systematic process that facilitates planning and design for dissemination, implementation and maintenance of EBls in practice. IM has been used to guide the design of many health interventions, focusing on program implementation. Less studied is its use to adapt and scale screening interventions within the healthcare clinic setting. This paper describes the development of an implementation intervention using $\mathrm{IM}$ to facilitate the adoption, implementation, and maintenance of an $\mathrm{EBI}$ designed to increase mammography adherence in healthcare clinics, the adapted Peace of Mind Program (PMP).

Methods: IM framework, Step 5, was used to guide the implementation intervention planning. IM guided identification of specific adoption, implementation, and maintenance performance objectives. We formed an implementation intervention planning group consisting of members of the academic team, our community partner and community health workers (CHWs) with substantial experience working on mammography screening programs in federally qualified health centers (FQHCs) and charity clinics.

Results: Results are presented by Intervention Mapping task for Step 5 (Program Implementation Plan). We describe how the consolidated framework for implementation research (CFIR) informed the selection of performance objectives, determinants, methods, and practical applications in the final implementation intervention.

Conclusions: This paper provides an example of the use of Intervention Mapping Step 5 and CFIR to create an implementation intervention to support EBI scale up of an evidence-based mammography intervention within a specific setting.

\section{Clinical trials registration number: NCT02296177}

Keywords: intervention mapping, implementation intervention, consolidated framework for implementation research, mammography, underserved women 


\section{BACKGROUND}

The research to practice gap is well-documented; only a fraction of evidence-based interventions (EBI) are integrated into practice settings and fewer still are sustained in practice over time (1-5). Although much work has begun to elucidate contextual factors influencing implementation, the specific processes that facilitate and hinder adoption, implementation, and maintenance of EBIs in clinical settings remains poorly understood $(6,7)$. Further, practitioners' knowledge and expertise is rarely effectively integrated into program design and testing, resulting in programs that may not fit well within the implementation context, or match the needs of the communities they were intended to benefit $(4,8-11)$. The development of effective implementation strategies should include participatory approaches and be guided by theory. Theory driven D\&I interventions that consider individual and systems-level change, can improve the likelihood of adoption, implementation and maintenance of EBIs (12) and support policy and practice changes that improve health outcomes over time. However, few programs to date have used theory to inform their approaches. Davies et al. reviewed 235 D\&I studies and found that only $23 \%$ used theory to inform the design of their strategies (13). Further, these D\&I strategies rarely use multi-level approaches to increase EBI use (14).

There are both few programs available that target mammography adherence in underserved populations specifically and even fewer that use well-defined adoption, implementation and sustainment interventions for mammography EBIs in the U.S. (15). Underserved populations (women who lack insurance or who are underinsured and low-income) have increased risk for late-stage breast cancer diagnosis due to a combination of factors, including lower mammography screening rates overall, high rates of missed screening appointments and lack of timely referral to diagnostic evaluation and treatment in those who screen abnormal (12). Considering the second factor (missing appointments), it has been shown that women who missed screening appointments were more likely to be diagnosed at a later stage of cancer than women who attended their appointments outside of the other two factors (16). This highlights the need for EBIs that improve mammography appointment attendance in underserved women since these women have already addressed the first step of engaging with the healthcare system and scheduling a screening appointment. Gaps in understanding of how best to translate lessons learned from research for integration of EBI's into everyday use-taking into account the local setting and needs of the multiple stakeholders has left many effective mammography programs unused or applied with limited fidelity (17).

Well-designed dissemination and implementation (D\&I) strategies are particularly important for the execution of multilevel interventions, which are typically used within complex practice systems such as health care settings to address differences in health outcomes (18). Intervention Mapping (IM) is a systematic process that facilitates planning and design for dissemination, implementation and maintenance of EBIs (19-22) in practice. Intervention mapping has been used to guide the design of many health interventions including a focus on program implementation (12). Less studied is its use to adapt and scale screening interventions within the healthcare clinic setting. This paper describes the development of an implementation intervention using Intervention Mapping to facilitate the adoption, implementation, and maintenance of an EBI designed to increase mammography adherence in healthcare clinics, the adapted Peace of Mind Program (PMP).

\section{METHODS}

PMP is a telephone-based EBI to increase mammography appointment adherence (attendance) in underserved women who have scheduled mammography screening appointments. PMP uses a scripted, tailored telephone counseling reminder call which was developed using the Transtheoretical Model of Change to counsel patients through barriers to appointment attendance, such as fear of screening or fear of outcome (4, $12,23,24)$. In addition, PMP engages the patient in active planning for their appointment, such as ensuring the correct paperwork has been completed and that required documents will be brought with the patient (e.g., proof of income) $(4,12$, $23,24)$. The PMP was designed for use in federally qualified health centers (FQHCs) and charity clinics providing access to mobile mammography services $(4,12,23,24)$. PMP had been previously adapted for underserved women and evaluated using IM [Int Map Adapt and found to effectively reduce appointment no-show rates from $44 \%$ (comparison) to $19 \%$ in the intervention arm $(23,24)$. The adjusted odds of a woman in the intervention group attending her appointment were 3.88. The adjusted odds of a woman attending her appointment in the intent-to-treat analysis were $2.31(23,24)]$. However, previous implementations of PMP lacked a mechanism for taking the program to scale across multiple sites. Our previous studies had focused on development and evaluation of program components, but had not focused on structures necessary to take the program to scale. For this project, our aim was to develop an implementation intervention to support the implementation and scale-up of the EBI in 20 FHQCs and charity clinics in the Greater Houston region, Texas (24). The Intervention Mapping framework, Step 5 was used to guide the implementation intervention planning. Intervention mapping guided identification of specific adoption, implementation, and maintenance performance objectives (who had to do what to implement the intervention). It helps the planning group identify determinants of implementation; why clinics (decision makers and staff) or clients would adopt, implement, and maintain the PMP (19).

IM allows for integration of theories and frameworks to inform the implementation intervention. In our project we used CFIR to inform the planning process. The CFIR is a meta-framework which includes five domains [intervention characteristics, outer setting, inner setting, characteristics of individuals and process; $(6,25)]$. Within these five domains are 39 underlying constructs that may influence implementation 
and development of clinical guidelines (6). CFIR was used to identify potential contextual factors that may influence the implementation and sustainability of the PMP as shown in Figure 1. Other theories that informed both the selection of determinants of implementations as well as methods for effecting change included Social Cognitive Theory, and Diffusion of Innovation $(26,27)$.

We formed an implementation intervention planning group to guide the process. The group consisted of members of the academic team, our community partner-the Breast Health Collaborative of Texas leadership and community health workers (CHWs) with substantial experience working on mammography screening programs in FQHCs and charity clinics in the Greater Houston area. Based on previous studies conducted by the team, the experience of planning team members in the community setting, and a review of the literature, we pre-determined that FQHCs and charity clinics were the primary stakeholders for adoption, implementation and maintenance. This project received approval from the Institutional Review Board at the academic institution, protocol number HSC-SPH-14-0269. Per Institutional Review Board review, written informed consent was not required. Women who later participated in the trial and received reminder phone calls gave verbal consent at the outset of the phone call.

\section{RESULTS OF THE APPLICATION OF IM STEP 5 FOR PMP DEVELOPMENT}

Results will be presented by Intervention Mapping task for Step 5 (Program Implementation Plan). We describe how CFIR informed the selection of performance objectives, determinants, methods, and practical applications included in the final implementation intervention.

\section{Task 1. Identify Program Adopters, Implementers and Maintainers}

We first identified what stakeholders would be involved in the adoption, implementation and maintenance of the PMP. We then held a brainstorming session with the planning group to answer key questions that would inform the development of the implementation intervention such as: (1) Who will make the decision to adopt the PMP program in FQHCs or community clinics and who will these decision-makers need to consult? (2) Who will implement the program? Will the PMP program require different people to implement different components? and (3) Who will ensure that the PMP program is maintained as long as it is needed? Following the brainstorming sessions, we completed detailed summaries to inform the following tasks and verified our implementers with clinic staff knowledge of FQHC and

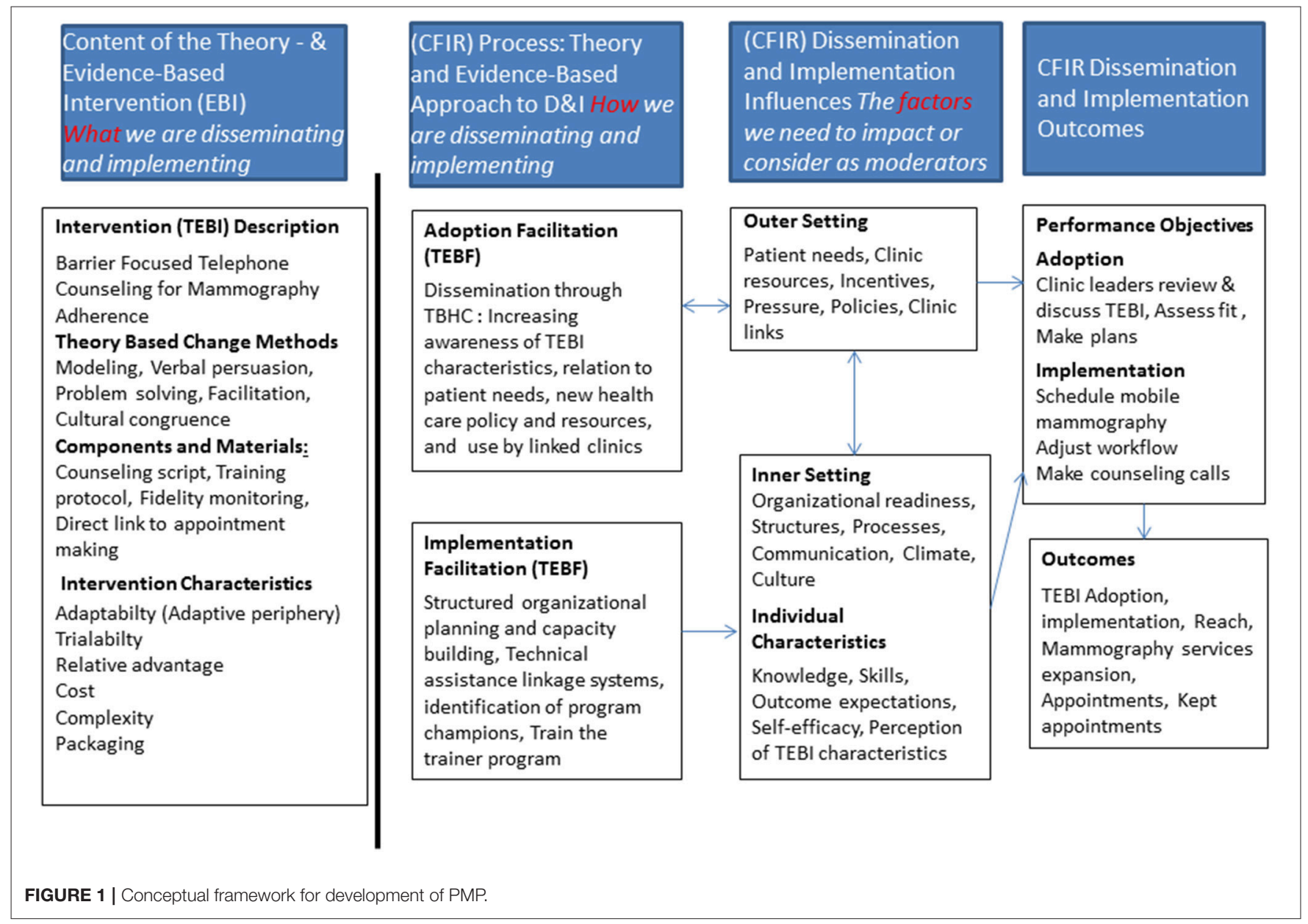




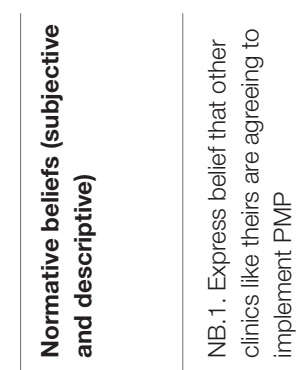

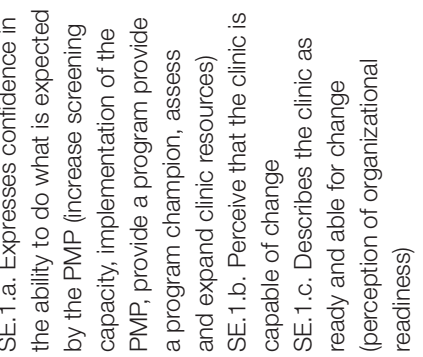
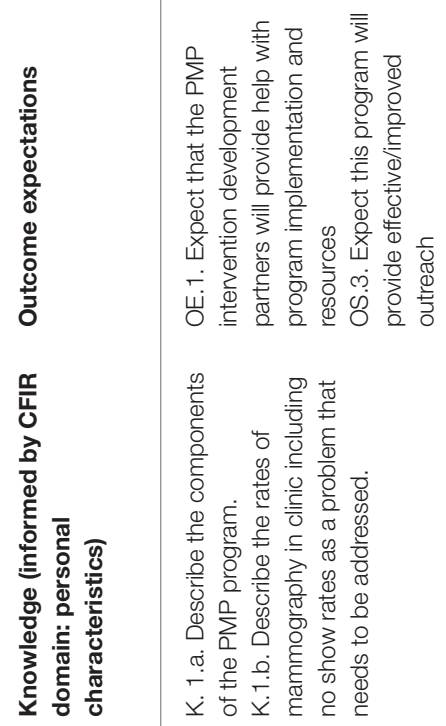
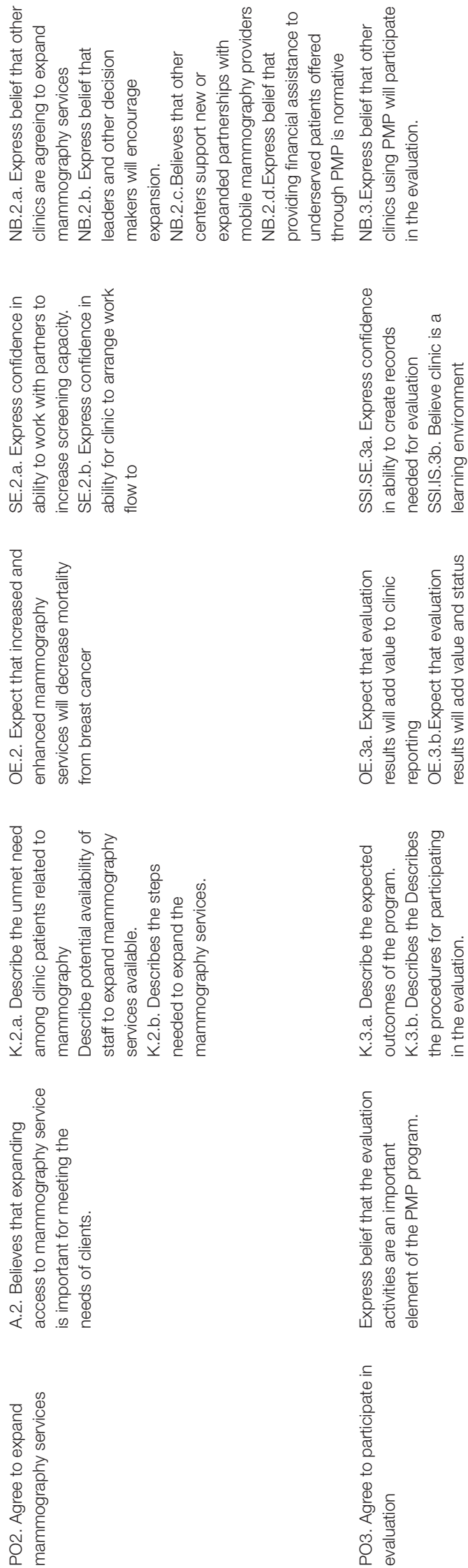
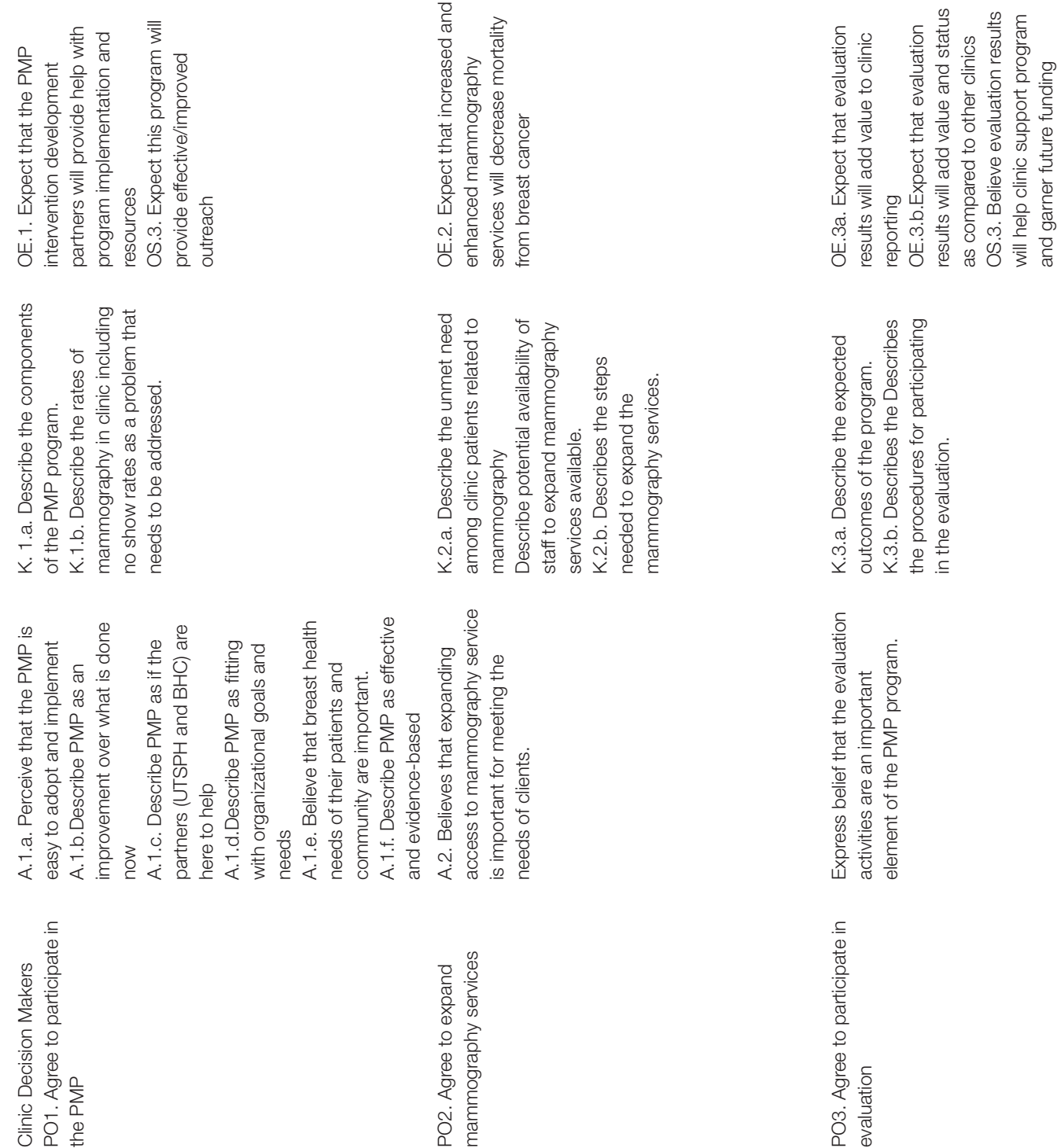

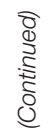




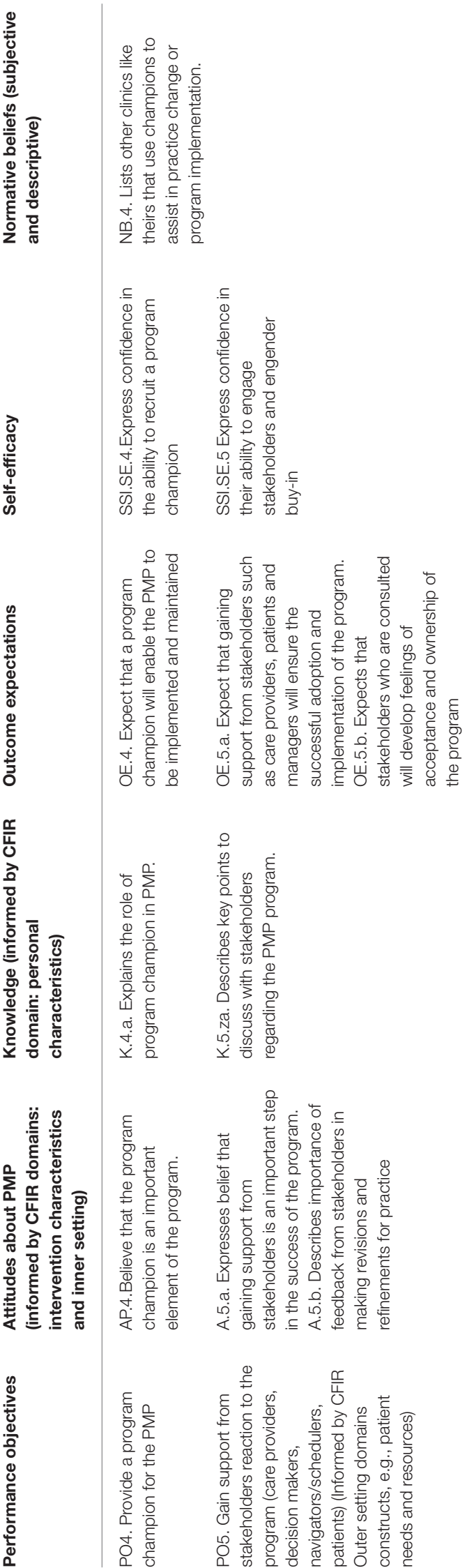

charity clinic structures. Based on our brainstorming sessions, the planning team determined that the clinic leader would be the adoption decision maker in our participating locations. Clinic leaders typically hold roles such as Executive Director or Chief Executive Officer and are decision makers. In order to adopt the program, the clinic leader would need to meet with the PMP team, review and sign an MOU and assign staff to participate in the program. Adoption performance objectives and determinants are summarized in Table $\mathbf{1 .}$

\section{Task 2. State Outcomes and Performance Objectives for Each Stage (Adoption, Implementation and Maintenance)}

For this task, the planning group sought to identify who needed to do what in order to adopt/implement/maintain the program. The planning group met and brainstormed answers to key questions such as: "What do FQHCs and charity clinics have to do in order to adopt PMP?" "What stakeholders does the planning group need to consult in order for PMP to be adopted?" "What levels of approval do the clinics need in order to adopt PMP?" To better understand and clearly articulate the goals for implementation, we posed the following questions: "What do the program implementers need to do to implement the essential PMP program components?" To better understand and articulate maintenance of PMP over time, we needed to more clearly understand what would be required to sustain the program in the clinics. Thus we posed the following questions: "What do they need to do to maintain the PMP program?" Our planning team held a brainstorming session and free-listed performance objectives for each. The CFIR domain "process of implementation" was useful in informing potential answers to this inquiry and subsequent selection of implementation performance objectives (what had to be done to implement the intervention).

In the brainstorming session, a facilitator led the team through answering each question and probed the planning group around specific constructs from CFIR and social cognitive theory to make sure the responses were also informed by theoretical and contextual consideration. We determined that the implementation for the EBI would be led by two clinic staff members, the mammography program manager (or clinic staff manager) and a community health worker/patient navigator based on clinic leadership and staff structure related to their overall environment and specifically to their mammography programs. Performance objectives and determinants for implementation are summarized in Table 2.

\section{Task 3. Create Matrices of Change Objectives}

The next task, development of matrices of change objectives, included the description of very specific objectives for adoption, implementation, and maintenance. First, we identified the determinants for each stage in a brainstorming session where the PMP planning team answered the following questions: "Why would adopters decide to use PMP?"; "Why would implementers do what is necessary to implement PMP?", and "Why would 


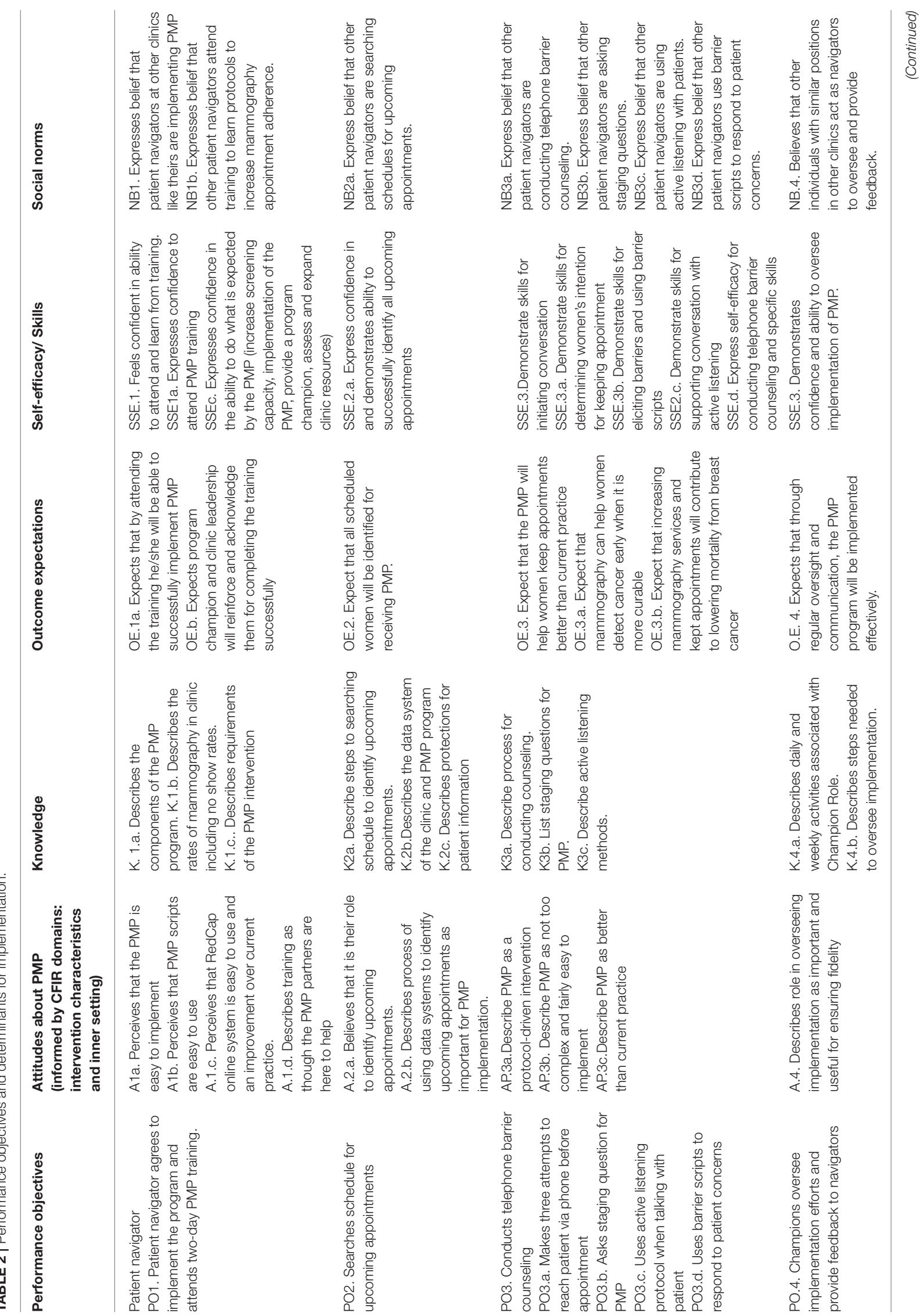




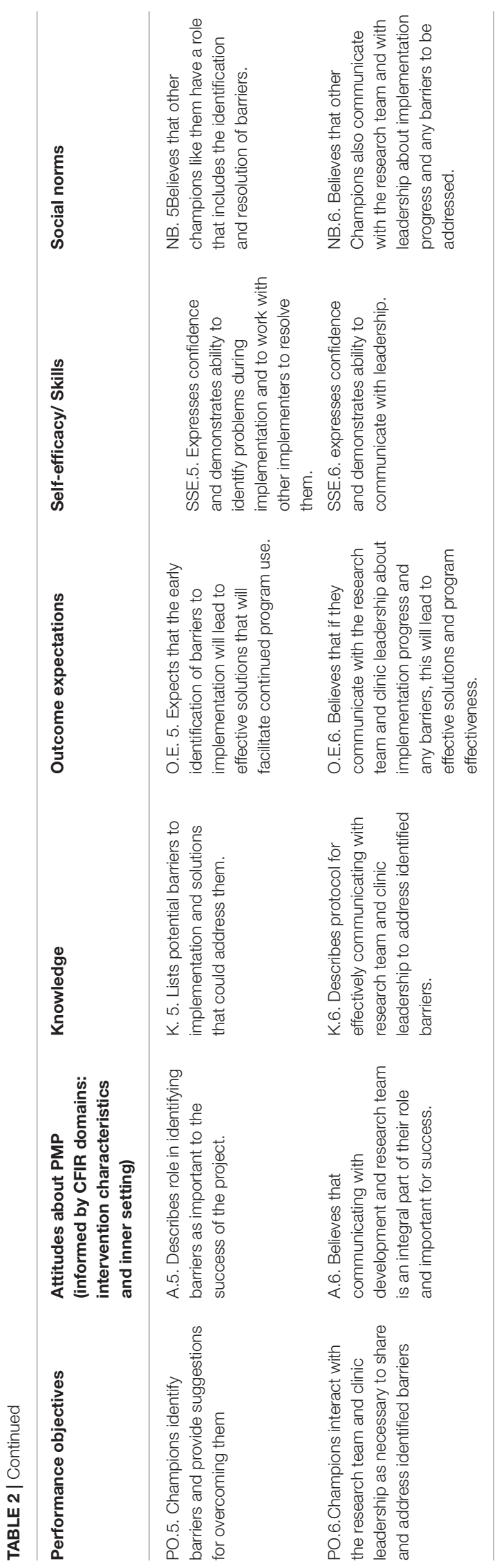

implementers of PMP do what it takes to make sure the program is continued over time?" The CFIR also informed the selection of determinants. For example, the CFIR domain, "characteristics of the innovation" (also describe in Diffusion of Innovation) informed the selection of specific attitudinal determinants that were expected to influence both adoption and implementation. These included attitudes about the efficacy, potential fit, and importance of the PMP program. Following the selection of determinants, we created the matrices of change objectives by crossing the identified determinants with performance objectives asking the question: what needs to change in the determinants (e.g., knowledge, skills) for the implementer to accomplish this performance objective. The resulting cells of the matrix represent specific change objectives that form the blueprint of the implementation intervention. The maintenance of the EBI program as practice would require a commitment from the clinic leadership, program manager and community health worker/patient navigator. The performance objectives and determinants for maintenance are summarized in Table 3.

\section{Task 4. Design Implementation Intervention Components}

The final task for IM for developing implementation interventions includes choosing the change methods and practical applications, designing the scope and sequence for program components and production of materials for influencing program use. The program planning group began this task by considering the determinants and list of change objectives created in Step 3. Next, they reviewed the relevant research and practice literature to confirm, refute, or modify the provisional list of change methods and their practical applications. This task was completed over a period of 2 months where the planning group met in bi-weekly sessions to review the outputs from Step 3, review and discuss the literature and iteratively update the list of change methods and practical applications. To guide our process, we used a combination of the theories diffusion of innovations $(28,29)$ and social cognitive theory. We were also guided by the constructs of the consolidated framework for implementation research (CFIR) as shown in Figure 1 through the selection of methods for the implementation intervention. We developed a PowerPoint presentation to keep the process organized which was updated at each team planning session and finalized. The presentation contained background information from the needs assessment, original program implementation and evaluation and brainstormed outcomes from each step of the IM process, serving as a complete record of project work which could be easily modified at each session and viewed by team members in remote locations (e.g., phone or internet connection).

The intervention change components (see Table 4), theoretical methods and practical applications for adoption, implementation and maintenance of the PMP program were developed to support the stated change objectives, including presentations, handbooks, training curricula, MOUs and newsletters. Examples of these program materials are available as a supplement to this article. Implementation of the program is 


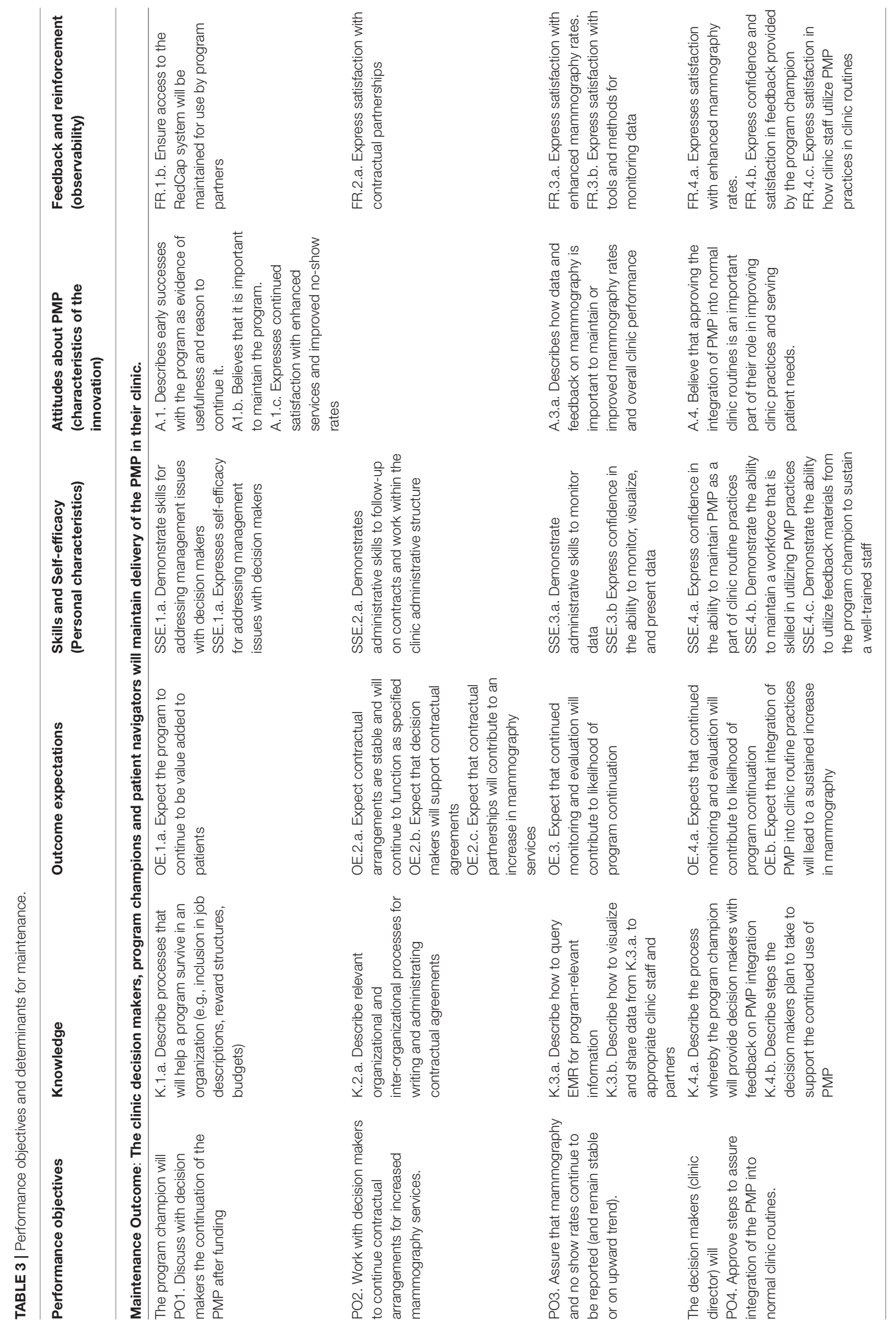


TABLE 4 | Peace of mind program implementation intervention plan.

\begin{tabular}{|c|c|c|c|c|}
\hline Stage & Agent & $\begin{array}{l}\text { Determinants/change } \\
\text { objectives }\end{array}$ & Theoretical change methods & Practical applications \\
\hline Adoption & Clinic Decision Maker & $\begin{array}{l}\text { Awareness/Perceptions of PMP } \\
\text { Outcome Expectations } \\
\text { Skills and Self-efficacy } \\
\text { Feedback and reinforcement }\end{array}$ & $\begin{array}{l}\text { PMP program information } \\
\text { Persuasion } \\
\text { Role Modeling }\end{array}$ & $\begin{array}{l}\text { Email blast to BHC members with PMP } \\
\text { informational video and link to pre-adoption survey } \\
\text { Webinar to BHC members covering } \\
\text { evidence-based approaches to breast cancer } \\
\text { prevention, PMP information and adoption steps } \\
\text { Adoption meeting held with interested clinics } \\
\text { Financial assistance to clinic } \\
\text { Assistance with connecting to mobile providers to } \\
\text { increase screening (as needed) }\end{array}$ \\
\hline Implementation & All & $\begin{array}{l}\text { Awareness/Perceptions } \\
\text { Outcome Expectations } \\
\text { Skills and Self-efficacy } \\
\text { Feedback and Reinforcement }\end{array}$ & $\begin{array}{l}\text { Cue to participate } \\
\text { Communication } \\
\text { Mobilization } \\
\text { Organizational Consultation/Planning }\end{array}$ & $\begin{array}{l}\text { Invite clinic staff to participate in stakeholder group } \\
\text { (templates for invitation email) } \\
\text { Email template for site visit (including requested } \\
\text { participants) and site visit questionnaire } \\
\text { Site visit planning meeting } \\
\text { Program implementation guide, clinic handbook, } \\
\text { stakeholder manual and computer assisted PMP } \\
\text { scripts reviewed during participatory stakeholder } \\
\text { meetings } \\
\text { Implementation readiness checklist } \\
\text { Stakeholder meetings to support implementation } \\
\text { (continue after reminder calls begin). E-newsletter } \\
\text { shared with stakeholders }\end{array}$ \\
\hline Implementation & $\begin{array}{l}\text { Program Champion } \\
\text { Navigator }\end{array}$ & $\begin{array}{l}\text { Awareness/Perceptions } \\
\text { Outcome Expectations } \\
\text { Skills and Self-efficacy } \\
\text { Feedback and Reinforcement }\end{array}$ & $\begin{array}{l}\text { Information } \\
\text { Persuasion } \\
\text { Skill building and guided practice } \\
\text { Modeling } \\
\text { Monitoring and feedback } \\
\text { Technical assistance/capacity building } \\
\text { Facilitation } \\
\text { Vicarious reinforcement }\end{array}$ & $\begin{array}{l}\text { Face to face training held over two } 4 \mathrm{~h} \text { sessions. } \\
\text { Training was submitted to Texas for CEU certification } \\
\text { for community health workers and social workers } \\
\text { BHC navigators model EBI behavior and provide } \\
\text { ongoing implementation support on-site } \\
\text { PMP research team available via email, phone and } \\
\text { training booster sessions as needed } \\
\text { Paperwork processes to provide funds for patients } \\
\text { needing financial assistance from PMP }\end{array}$ \\
\hline Maintenance & $\begin{array}{l}\text { Program Champion } \\
\text { Decision Makers }\end{array}$ & $\begin{array}{l}\text { Outcome Expectations } \\
\text { Skills and Self-efficacy } \\
\text { Feedback and Reinforcement }\end{array}$ & $\begin{array}{l}\text { Information } \\
\text { Persuasion } \\
\text { Technical assistance }\end{array}$ & $\begin{array}{l}\text { Face to face meeting to discuss maintaining } \\
\text { program } \\
\text { Program wrap up email with instructions for } \\
\text { continued access to program scripts and contact } \\
\text { info for technical support } \\
\text { Continued access to online PMP scripts } \\
\text { Technical support as needed via email or phone }\end{array}$ \\
\hline
\end{tabular}

supported through the use of a participatory stakeholder group, where clinic program staff participated in regular meetings with the PMP team to review program materials, address any needed adaptations and timeline adjustments, train in the use of the PMP scripts and online system, phase in implementation (clinic staff slowly take over ownership of the reminder phone calls) and ensure active troubleshooting of any program issues during implementation. Implementation is also supported through the use of bilingual community health workers and PMP materials which are available in multiple languages. Through the site visit, PMP staff collect information on language needs of program participants and translate materials accordingly. Implementation is also supported through the use of an online interface programmed in REDCap which guides the community health worker through each patient phone call starting from informed consent and through all intervention components. Using a simple interface, the community health worker is guided through the scripted intervention and advances to the next step by completing either pre-programmed check boxes or openended text boxes. The system collects data on informed consent, the patient's stage of readiness to attend their appointment, barriers counseled and logistical planning offered during the phone call.

The PMP was implemented over the course of three phases in each clinic. In phase one, the following steps were accomplished: (1) We conducted site assessments with each clinic to understand baseline processes in their mammography programs, (2) Clinic staff were invited to join the participatory stakeholder group, (3) Stakeholder meetings began and reviewed program materials and recommended adaptations to the implementation protocol as needed, (4) PMP training takes place, and (5) Implementation checklist is used to ensure readiness to start PMP phone calls. In phase two, the following steps are accomplished: (1) BHC navigators on-site, provide role modeling of phone calls and support clinic staff as the program begins, (2) Navigators transition reminder phone call scheduling and responsibility to 
clinic staff over a period of several months and then monitor calls to ensure fidelity, (3) Re-training is provided as needed during this phase and (4) Stakeholder meetings continue with a focus on troubleshooting any implementation barriers and creating e-newsletters to re-inforce program behaviors and highlight program successes. In phase three, clinics take more responsibility for the program and $\mathrm{BHC}$ navigators reduce on-site monitoring. Troubleshooting of implementation issues continues. Finally, as clinics move to maintenance, the PMP team holds a meeting with clinic leadership to discuss PMP maintenance plans and provides information on continued access to the PMP online system and technical support. To support maintenance, the PMP online system remains available to all participating clinics.

\section{DISCUSSION}

Poor rates of EBI adoption and low levels of implementation and maintenance, may lead to ineffective or less than expected impact on health poor outcomes when translating EBIs to practice in the community (19). The research to practice gap will persist until successful models are developed to support adoption, implementation and maintenance of EBIs within real-world settings. Practitioners and investigators have called for better descriptions of the development of implementation interventions to facilitate replication and refinement of EBI implementations and dissemination (30-32). However, there are few published studies which provide information on the process used to develop implementation interventions or how implementation science frameworks, such as CFIR, can inform implementation intervention planning $(14,31,33)$. Neta et al. (22) note that despite many calls for research showing the process or frameworks used to develop implementation interventions, it is typically not at all apparent how planners took these issues under consideration while planning their programs (e.g., the "what" and "how") (22). The authors further note that systematic approaches, including IM, could address this need, especially when used in conjunction with theory (22). This paper provides an example of the use of Intervention Mapping Step 5 and CFIR to create an implementation intervention to support EBI scale up of an evidence-based mammography intervention within a specific setting (FQHCs and charity clinics). A recent systematic review of studies using CFIR found only two that had fully used the CFIR in the pre-implementation phase (34). We found that the inclusion of CFIR determinants in the planning process can facilitate critical implementation intervention design and development, increasing the likelihood of successful dissemination and implementation (34). The development of the implementation intervention resulted in the identification of key determinants that we then created specific strategies and methods for addressing through training and targeted messaging for adopters, implementers and for promotion of program maintenance. We further hypothesized that the use of a participatory stakeholder group would support implementation based both on our conceptual framework and from discussions in the brainstorming sessions about clinics' need for implementation support and to help ensure fidelity of implementation. One of the limitations to our participatory approach was balancing the amount of time required from our community partners to participate in brainstorming and planning sessions. Our team addressed this challenge by focusing our time with community partners on brainstorming activities. We spent additional time outside these meeting sessions working on transcription and translation of the brainstorming materials into EBI components which were then reviewed by our community members. An additional challenge was in getting all levels of FQHC and clinic staff to participate in these planning sessions. Clinic staff have many responsibilities and are not always able to take time away from the office for planning meetings, especially unpaid. We addressed this by working with a community partner who was knowledge of our local clinics and with community health workers who had previously worked in a number of the local clinics. In an ideal setting, we would have had clinic leadership directly participate in the planning sessions.

The Peace of Mind Program developed in this project was adapted from an existing EBI and previously tailored to our local community context for specific mammography barriers. In evaluating the EBI effectiveness, the implementation of the program had been highly tailored to that environment. Further, based on our knowledge of FQHCs and charity clinics in the Greater Houston area, we knew there was heterogeneity in the clinic environments, staffing and mammography program processes. Therefore, we hypothesized that adding a structured, theory-based implementation intervention more broadly relevant to FQHCs and charity clinics to the EBI would be necessary for successful scale-up within this specific context. A recent systematic review of the research-practice gap in primary care settings supports this hypothesis. Lau et al. (35) found overlap with EBI adoption, implementation and maintenance and CFIR constructs used in this project to develop the implementation intervention (what Lau et al. refer to as contextual factors). Additionally, the review highlighted that these "contextual factors" are often notably absent from research and frequently fail to be acknowledged, described or taken into account during implementation or program planning (35). The PMP is currently being evaluated using a non-randomized controlled stepped wedge trial in 16 FQHCs and charity clinics in the Greater Houston area. IM Step 6 was used to guide the development of the evaluation plan for PMP, including measures specific to adoption and implementation within clinics participating in the trial. A full description of the development of the evaluation plan for PMP is beyond the scope of this manuscript. For further information on the evaluation of PMP, we refer readers to Highfield et al. (24), which details the protocol for the PMP trial (24). Briefly, reach of PMP is being measured using Google Analytics tracking from BHC outreach events described above (e.g., email communications, webinars) and through the collection of participation logs for events. Adoption and implementation are being measured through the use of a validated survey of CFIR constructs which was adapted for this project. Implementation is also being measured through our REDCap interface, which tracks navigator's use of the EBI staging question. Evaluation results from the trial are expected in late 
2018 and will be able to provide further insight into the effect of our implementation intervention on program implementation, maintenance, fidelity and outcomes (appointment attendance). While full evaluation of the program is underway and will be reported elsewhere, a total of 16 clinics with 24 operating sites, providing mammography services to over 4,500 women during our project period adopted PMP. These clinics served a diverse population of Caucasian, Hispanic, African American and Vietnamese women (all underserved). We anticipate that the program will lead to an increase in mammography screening in participating clinics as a result of the EBI components focused on assessing current screening goals, relationships with mobile providers and serving as a bridge between clinics and providers. Increases in screening in underserved women are important as screening serves as the first step in the pathway to breast cancer disparities (16).

In addition, we are monitoring appointment adherence (no-show rates) along with appointment cancellations, reschedules, patients turned away for incomplete paperwork and other reasons why a woman may not ultimately complete her mammogram appointment. We believe that assessing the EBI against these additional factors will provide a more complete picture of screening outcomes and barriers. To our knowledge, this is the only paper to date which has applied Intervention Mapping in conjunction with the constructs of the CFIR framework and behavioral theories to develop a systematic implementation intervention for the scale up of a mammography EBI in FQHCs and charity clinics. While this paper is focused specifically on mammography screening, the approach we designed for implementation and the protocols and program materials could serve as a guide for others interested in developing similar programs.

\section{CONCLUSIONS}

EBIs which are tested and available for scale up may benefit from use of a structured implementation intervention process. In addition, this paper may provide useful insights for others

\section{REFERENCES}

1. Brownson RC, Baker EA, Deshpande AD, Gillespie KN. Evidence-Based Public Health. New York, NY: Oxford University Press (2017).

2. Brownson RC, Fielding JE, Maylahn CM. Evidence-based public health: a fundamental concept for public health practice. Annu Rev Public Health. (2009) 30:175-201. doi: 10.1146/annurev.publhealth.031308.100134

3. Chaudoir SR, Dugan AG, Barr CH. Measuring factors affecting implementation of health innovations: a systematic review of structural, organizational, provider, patient, and innovation level measures. Implement Sci. (2013) 8:22. doi: 10.1186/1748-5908-8-22

4. Highfield L, Bartholomew LK, Hartman MA, Ford MM, Balihe P. Grounding evidence-based approaches to cancer prevention in the community: a case study of mammography barriers in underserved African American women. Health Promot Pract. (2014) 15:904-14. doi: 10.1177/1524839914534685

5. Burgio LD. Disentangling the translational sciences: a social science perspective. Res Theory Nurs Pract. (2010) 24:56-63. doi: 10.1891/1541-6577.24.1.56 interested in bridging CFIR, health behavioral theories and scaling up EBIs in community settings, particularly those related to mammography screening in healthcare settings and will facilitate use of the IM steps to support the systematic review and addressing of context specific needs for adoption, implementation and maintenance of EBIs into practice.

\section{ETHICS STATEMENT}

This study was carried out in accordance with the recommendations of UTHealth Committee for the Protection of Human Subjects. The protocol was approved by the UTHealth Committee for the Protection of Human Subjects. All subjects gave verbal informed consent in accordance with the Declaration of Helsinki.

\section{AUTHOR CONTRIBUTIONS}

LH conceptualized the study, carried out the study and lead the writing of the article. LE-B conceptualized and served as the mentor for the study. MV and MF assisted with study development and manuscript writing.

\section{FUNDING}

This project was funded under contract/grant number R18HS023255-03 from the Agency for Healthcare Research and Quality (AHRQ), U.S. Department of Health and Human Services. The opinions expressed in this document are those of the authors and do not reflect the official position of AHRQ or the U.S. Department of Health and Human Services.

\section{ACKNOWLEDGMENTS}

The authors would like to acknowledge our community partner, the Breast Health Collaborative of Texas and our mobile mammography partners, The Rose, MD Anderson Cancer Center and Mammosafe.
6. Damschroder LJ, Aron DC, Keith RE, Kirsh SR, Alexander JA, Lowery JC. Fostering implementation of health services research findings into practice: a consolidated framework for advancing implementation science. Implement Sci. (2009) 4:50. doi: 10.1186/1748-5908-4-50

7. Wandersman A, Duffy J, Flaspohler P, Noonan R, Lubell K, Stillman L, et al. Bridging the gap between prevention research and practice: the interactive systems framework for dissemination and implementation. Am J Community Psychol. (2008) 41:171-81. doi: 10.1007/s10464-008-9174-z

8. Kessler R, Glasgow RE. A proposal to speed translation of healthcare research into practice: dramatic change is needed. Am J Prev Med. (2011) 40:637-44. doi: 10.1016/j.amepre.2011.02.023

9. Kerner JF. Integrating research, practice, and policy: what we see depends on where we stand. J Public Health Manage Pract. (2008) 14:193-8. doi: 10.1097/01.PHH.0000311899.11197.db

10. Ramanadhan S, Crisostomo J, Alexander-Molloy J, Gandelman E, Grullon M, Lora V, et al. Perceptions of evidence-based programs among communitybased organizations tackling health disparities: a qualitative study. Health Educ Res. (2011) 27:717-28. doi: 10.1093/her/cyr088 
11. Stetler CB, McQueen L, Demakis J, Mittman BS. An organizational framework and strategic implementation for system-level change to enhance research-based practice: QUERI Series. Implement Sci. (2008) 3:30. doi: $10.1186 / 1748-5908-3-30$

12. Highfield L, Hartman MA, Mullen PD, Rodriguez SA, Fernandez ME, Bartholomew LK. Intervention mapping to adapt evidencebased interventions for use in practice: increasing mammography among African American women. Biomed Res Int. (2015) 2015:160103. doi: $10.1155 / 2015 / 160103$

13. Davies P, Walker AE, Grimshaw JM. A systematic review of the use of theory in the design of guideline dissemination and implementation strategies and interpretation of the results of rigorous evaluations. Implement Sci. (2010) 5:14. doi: 10.1186/1748-5908-5-14

14. Proctor EK, Powell BJ, McMillen JC. Implementation strategies: recommendations for specifying and reporting. Implement Sci. (2013) 8:139. doi: 10.1186/1748-5908-8-139

15. Byers TE, Wolf HJ, Bauer KR, Bolick-Aldrich S, Chen VW, Finch JL, et al. The impact of socioeconomic status on survival after cancer in the United States. Cancer. (2008) 113:582-91. doi: 10.1002/cncr.23567

16. Onitilo AA, Engel JM, Liang H, Stankowski RV, Miskowiak DA, Broton M, et al. Mammography utilization: patient characteristics and breast cancer stage at diagnosis. Am J Roentgenol. (2013) 201:1057-63. doi: 10.2214/AJR.13.10733

17. Yano EM, Green LW, Glanz K, Ayanian JZ, Mittman BS, Chollette V, et al. Implementation and spread of interventions into the multilevel context of routine practice and policy: implications for the cancer care continuum. J Natl Cancer Inst Monogr. (2012) 2012:86-99. doi: 10.1093/jncimonographs/lgs004

18. Watson DP, Young J, Ahonen E, Xu H, Henderson M, Shuman V, et al. Development and testing of an implementation strategy for a complex housing intervention: protocol for a mixed methods study. Implement Sci. (2014) 9:138. doi: 10.1186/s13012-014-0138-4

19. Eldredge LKB, Markham CM, Ruiter RA, Kok G, Parcel GS. Planning Health Promotion Programs: An Intervention Mapping Approach. San Francisco, CA: John Wiley \& Sons (2016).

20. Brownson RC, Jacobs JA, Tabak RG, Hoehner CM, Stamatakis KA. Designing for dissemination among public health researchers: findings from a national survey in the United States. Am J Public Health. (2013) 103:1693-9. doi: 10.2105/AJPH.2012.301165

21. Harris JR, Cheadle A, Hannon PA, Lichiello P, Forehand M, Mahoney E, et al. A framework for disseminating evidence-based health promotion practices. Prev Chronic Dis. (2012) 9:110081. doi: 10.5888/pcd9.110081

22. Neta G, Glasgow RE, Carpenter CR, Grimshaw JM, Rabin BA, Fernandez $\mathrm{ME}$, et al. A framework for enhancing the value of research for dissemination and implementation. Am J Public Health. (2015) 105:49-57. doi: 10.2105/AJPH.2014.302206

23. Highfield L, Hartman MA, Bartholomew LK, Balihe P, Ausborn VA. Evaluation of the effectiveness and implementation of an adapted evidencebased mammography intervention for African American women. Biomed Res Int. (2015) 2015:240240. doi: 10.1155/2015/240240
24. Highfield L, Rajan SS, Valerio MA, Walton G, Fernandez ME, Bartholomew LK. A non-randomized controlled stepped wedge trial to evaluate the effectiveness of a multi-level mammography intervention in improving appointment adherence in underserved women. Implement Sci. (2015) 10:143. doi: 10.1186/s13012-015-0334-x

25. Damschroder LJ, Hagedorn HJ. A guiding framework and approach for implementation research in substance use disorders treatment. Psychol Addict Behav. (2011) 25:194. doi: 10.1037/a0022284

26. Bandura A. Social Foundation of Thought and Action: A Social-Cognitive View. Englewood Cliffs, NJ: Prentice-Hall (1986).

27. Bandura A. Social cognitive theory: an agentic perspective. Annu Rev Psychol. (2001) 52:1-26. doi: 10.1146/annurev.psych.52.1.1

28. Rogers EM. Diffusion of Innovations. 3rd ed. New York, NY: Free Press (1983).

29. Rogers EM. Diffusion of Innovations. 4th ed. New York, NY: Free Press (1995)

30. Abraham C, Albarracín D, Araújo-Soares V. WIDER Recommendations to Improve Reporting of the Content of Behaviour Change Interventions. Abraham C. (2009).

31. Hoffmann TC, Erueti C, Glasziou PP. Poor description of nonpharmacological interventions: analysis of consecutive sample of randomised trials. BMJ (2013) 347:f3755. doi: 10.1136/bmj.f3755

32. Hoffmann TC, Glasziou PP, Boutron I, Milne R, Perera R, Moher D, et al. Better reporting of interventions: template for intervention description and replication (TIDieR) checklist and guide. BMJ (2014) 348:g1687. doi: 10.1136/bmj.g1687

33. Lloyd JJ, Logan S, Greaves CJ, Wyatt KM. Evidence, theory and contextusing intervention mapping to develop a school-based intervention to prevent obesity in children. Int J Behav Nutr Phys Activity (2011) 8:73. doi: 10.1186/1479-5868-8-73

34. Kirk MA, Kelley C, Yankey N, Birken SA, Abadie B, Damschroder L. A systematic review of the use of the Consolidated Framework for Implementation Research. Implement Sci. (2016) 11:72. doi: 10.1186/s13012-016-0437-z

35. Lau R, Stevenson F, Nio B, Ong K, Dziedzic ST, Eldridge S, et al. Achieving change in primary care-causes of the evidence to practice gap: systematic reviews of reviews. Implement Sci. (2016) 11:40. doi: 10.1186/s13012-016-0396-4

Conflict of Interest Statement: The authors declare that the research was conducted in the absence of any commercial or financial relationships that could be construed as a potential conflict of interest.

Copyright (C) 2018 Highfield, Valerio, Fernandez and Eldridge-Bartholomew. This is an open-access article distributed under the terms of the Creative Commons Attribution License (CC BY). The use, distribution or reproduction in other forums is permitted, provided the original author(s) and the copyright owner(s) are credited and that the original publication in this journal is cited, in accordance with accepted academic practice. No use, distribution or reproduction is permitted which does not comply with these terms. 